\title{
OR7-003 - MEFV genotype, IL1B and role of NLRP3 in FMF
}

\author{
A Omenetti ${ }^{1,2^{*}}$, S Carta ${ }^{3}$, L Delfino ${ }^{3}$, A Martini $^{1,2}$, M Gattorno ${ }^{1}$, A Rubartelli $^{3}$ \\ From 7th Congress of International Society of Systemic Auto-Inflammatory Diseases (ISSAID) \\ Lausanne, Switerland. 22-26 May 2013
}

\begin{abstract}
Introduction
Familial Mediterranean fever (FMF) is the most common of the hereditary autoinflammatory disorders. FMF is caused by mutations of $M E F V$ gene which encodes for pyrin. It has been recently reported that frequency of FMF-like symptoms decreases from patients carrying two high penetrance mutations towards patients with a single low penetrance mutation. The effectiveness of interleukin (IL)-1b blockers has suggested that IL-1b may play a role in the pathophysiology of the disease. However, evidence of dysregulated IL-1b secretion in FMF patients is so far missing. Moreover, the role of NLRP3 has never been directly examined in FMF patients.
\end{abstract}

\section{Objectives}

To define in patients affected by Familial Mediterranean Fever (FMF), whether or not interleukin (IL)- $1 \beta$ secretion (1) is enhanced, (2) correlates with the type of MEFV mutation and (3) is mediated by NLRP3.

\section{Methods}

Freshly isolated monocytes from 20 FMF patients (12 homozygous and 8 heterozygous), 14 MEFV healthy carriers (HC) and 30 healthy donors (HD), unstimulated or after LPS-induced activation, were analyzed for redox state (reactive oxygen species (ROS) production and antioxidant responses), and for IL- $1 \beta$ and IL-1Receptor antagonist (IL-1Ra) secretion. NLRP3 down-modulation was induced by NLRP3 in vitro silencing.

\section{Results}

LPS-stimulated monocytes from FMF patients displayed enhanced IL-1 $\beta$ secretion which correlated with the number and penetrance of MEFV mutations. Silencing of NLRP3 consistently inhibited IL-1 $\beta$ secretion. As in other autoinflammatory diseases, MEFV mutated monocytes produced more ROS than genetically negative controls. However, contrary to CAPS, they were featured by a conserved and sustained antioxidant response. Consistent with this finding, MEFV mutated monocytes did not exhibit the functional indicators of oxidative stress observed in CAPS, including accelerated IL- $1 \beta$ secretion and deficient IL-1Ra production.

\section{Conclusion}

MEFV mutated monocytes display enhanced IL-1 $\beta$ secretion which correlates with the number of highpenetrance mutations and level of endogenous ROS. Unlike NLRP3 mutated cells, monocytes carrying MEFV mutations withstand oxidative stress and preserve IL-1Ra production, thereby limiting inflammation. Finally, in contrast to what found in the animal model, the increased secretion of IL- $1 \beta$ by LPS-stimulated FMF monocytes is NLRP3-dependent.

\section{Disclosure of interest}

None declared.

\section{Authors' details}

${ }^{1} \cup O$ Pediatria II, G Gaslini IRCCS, Genoa, Italy. ${ }^{2}$ University of Genoa, Genoa, Italy. ${ }^{3}$ UO Cell Biology, IRCCS AOU San Martino - IST, Genoa, Italy.

Published: 8 November 2013

doi:10.1186/1546-0096-11-S1-A104

Cite this article as: Omenetti et al:: OR7-003 - MEFV genotype, IL1B and role of NLRP3 in FMF. Pediatric Rheumatology 2013 11(Suppl 1):A104.

${ }^{1}$ UO Pediatria II, G Gaslini IRCCS, Genoa, Italy

Full list of author information is available at the end of the article 